\title{
On Poly-Bernoulli polynomials of the second kind with umbral calculus viewpoint
}

\author{
Dae San Kim ${ }^{1}$, Taekyun Kim² ${ }^{2 *}$ Toufik Mansour ${ }^{3}$ and Dmitry V Dolgy ${ }^{4}$
}

${ }^{*}$ Correspondence: tkkim@kw.ac.kr ${ }^{2}$ Department of Mathematics, Kwangwoon University, Seoul, South Korea

Full list of author information is available at the end of the article

\begin{abstract}
Poly-Bernoulli polynomials of the second kind were introduced in Kim et al. (Adv. Differ. Equ. 2014:219, 2014) as a generalization of the Bernoulli polynomial of the second kind. Here we investigate those polynomials and derive further results about them by using umbral calculus.
\end{abstract}

Keywords: Bernoulli polynomials; poly-Bernoulli polynomials; Stirling numbers; umbral calculus

\section{Introduction}

Following Kaneko [1], the poly-Bernoulli polynomials have been studied by many researchers in recent decades. Poly-Bernoulli polynomials $B_{n}^{(k)}(x)$ were defined as $\frac{L i_{k}\left(1-e^{-t}\right)}{1-e^{-t}} e^{x t}=\sum_{n \geq 0} B_{n}^{(k)}(x) \frac{t^{n}}{n !}$, where $L i_{k}(x)=\sum_{r \geq 1} \frac{x^{r}}{r^{k}}$ is the classical polylogarithm function, which satisfies $\frac{d}{d x} L i_{k}(x)=\frac{1}{x} L i_{k-1}(x)$. The poly-Bernoulli polynomials have wideranging applications in mathematics and applied mathematics (see [2-4]). For $k \in \mathbb{Z}$, the poly-Bernoulli polynomials $b_{n}^{(k)}(x)$ of the second kind are given by the generating function

$$
\frac{L i_{k}\left(1-e^{-t}\right)}{\log (1+t)}(1+t)^{x}=\sum_{n \geq 0} b_{n}^{(k)}(x) \frac{t^{n}}{n !}
$$

When $x=0, b_{n}^{(k)}=b_{n}^{(k)}(0)$ are called the poly-Bernoulli numbers of the second kind. When $k=1, b_{n}(x)=b_{n}^{(1)}(x)$ are called the Bernoulli polynomial of the second kind (see [5-11]). Poly-Bernoulli polynomials of the second kind were introduced as a generalization of the Bernoulli polynomial of the second kind (see [12]). The aim of this paper is to use umbral calculus to obtain several new and interesting explicit formulas, recurrence relations and identities of poly-Bernoulli polynomials of the second kind. Umbral calculus has been used in numerous problems of mathematics. Umbral techniques have been of use in different areas of physics; for example it is used in group theory and quantum mechanics by Biedenharn et al. (see [13-15]).

Let $\Pi$ be the algebra of polynomials in a single variable $x$ over $\mathbb{C}$ and let $\Pi^{*}$ be the vector space of all linear functionals on $\Pi$. We denote the action of a linear functional $L$ on a polynomial $p(x)$ by $\langle L \mid p(x)\rangle$. Define the vector space structure on $\Pi^{*}$ by $\left\langle c L+c^{\prime} L^{\prime} \mid p(x)\right\rangle=$ $c\langle L \mid p(x)\rangle+c^{\prime}\left\langle L^{\prime} \mid p(x)\right\rangle$, where $c, c^{\prime} \in \mathbb{C}$ (see [16-19]). We define the algebra of a formal power 
series in a single variable $t$ to be

$$
\mathcal{H}=\left\{f(t)=\sum_{k \geq 0} a_{k} \frac{t^{k}}{k !} \mid a_{k} \in \mathbb{C}\right\}
$$

The formal power series in the variable $t$ defines a linear functional on $\Pi$ by setting $\left\langle f(t) \mid x^{n}\right\rangle=a_{n}$, for all $n \geq 0$ (see [16-19]). Thus

$$
\left\langle t^{k} \mid x^{n}\right\rangle=n ! \delta_{n, k}, \quad \text { for all } n, k \geq 0 \text { (see [16-19]), }
$$

where $\delta_{n, k}$ is the Kronecker symbol. Let $f_{L}(t)=\sum_{n \geq 0}\left\langle L \mid x^{n}\right\rangle \frac{t^{n}}{n !}$. By (1.3), we have $\left\langle f_{L}(t) \mid x^{n}\right\rangle=$ $\left\langle L \mid x^{n}\right\rangle$. Thus, the map $L \mapsto f_{L}(t)$ is a vector space isomorphism from $\Pi^{*}$ onto $\mathcal{H}$. Therefore, $\mathcal{H}$ is thought of as a set of both formal power series and linear functionals. We call $\mathcal{H}$ the umbral algebra. The umbral calculus is the study of the umbral algebra.

Let $f(t)$ be a non-zero power series, the order $O(f(t))$ is the smallest integer $k$ for which the coefficient of $t^{k}$ does not vanish. If $O(f(t))=1$ (respectively, $\left.O(f(t))=0\right)$, then $f(t)$ is called a delta (respectively, an invertable) series. Suppose that $f(t)$ is a delta series and $g(t)$ is an invertable series, then there exists a unique sequence $s_{n}(x)$ of polynomials such that $\left\langle g(t)(f(t))^{k} \mid s_{n}(x)\right\rangle=n ! \delta_{n, k}$, where $n, k \geq 0$. The sequence $s_{n}(x)$ is called the Sheffer sequence for $(g(t), f(t))$ which is denoted by $s_{n}(x) \sim(g(t), f(t))$ (see $\left.[18,19]\right)$. For $f(t) \in \mathcal{H}$ and $p(x) \in$ $\Pi$, we have $\left\langle e^{y t} \mid p(x)\right\rangle=p(y),\langle f(t) g(t) \mid p(x)\rangle=\langle g(t) \mid f(t) p(x)\rangle$, and $f(t)=\sum_{n \geq 0}\left\langle f(t) \mid x^{n}\right\rangle \frac{t^{n}}{n !}$ and $p(x)=\sum_{n \geq 0}\left\langle t^{n} \mid p(x)\right\rangle \frac{x^{n}}{n !}($ see $[18,19])$. Thus, we obtain $\left\langle t^{k} \mid p(x)\right\rangle=p^{(k)}(0)$ and $\left\langle 1 \mid p^{(k)}(x)\right\rangle=$ $p^{(k)}(0)$, where $p^{(k)}(0)$ denotes the $k$ th derivative of $p(x)$ with respect to $x$ at $x=0$. Therefore, we get $t^{k} p(x)=p^{(k)}(x)=\frac{d^{k}}{d x^{k}} p(x)$, for all $k \geq 0$ (see $\left.[18,19]\right)$. Thus, for $s_{n}(x) \sim(g(t), f(t))$, we have

$$
\frac{1}{g(\bar{f}(t))} e^{y \bar{f}(t)}=\sum_{n \geq 0} s_{n}(y) \frac{t^{n}}{n !}
$$

for all $y \in \mathbb{C}$, where $\bar{f}(t)$ is the compositional inverse of $f(t)$ (see [18, 19]). For $s_{n}(x) \sim$ $(g(t), f(t))$ and $r_{n}(x) \sim(h(t), \ell(t))$, let $s_{n}(x)=\sum_{k=0}^{n} c_{n, k} r_{k}(x)$, then we have

$$
c_{n, k}=\frac{1}{k !}\left\langle\frac{h(\bar{f}(t))}{g(\bar{f}(t))}(\ell(\bar{f}(t)))^{k} \mid x^{n}\right\rangle
$$

(see $[18,19])$.

It is immediate from (1.1) and (1.4) to see that $b_{n}^{(k)}(x)$ is the Sheffer polynomial for the pair $g(x)=\frac{t}{L i_{k}\left(1-e^{1-e^{t}}\right)}$ and $f(t)=e^{t}-1$, that is,

$$
b_{n}^{(k)}(x) \sim\left(\frac{t}{L i_{k}\left(1-e^{1-e^{t}}\right)}, e^{t}-1\right) .
$$

The aim of the present paper is to present several new identities for the poly-Bernoulli polynomials by the use of umbral calculus. 


\section{Explicit expressions}

Before proceeding, we observe that

$$
\begin{aligned}
L i_{k}\left(1-e^{-t}\right) & =\sum_{r \geq 1} \frac{1}{r^{k}}\left(1-e^{-t}\right)^{r}=\sum_{r \geq 1} \frac{(-1)^{r}}{r^{k}}\left(e^{-t}-1\right)^{r} \\
& =\sum_{r \geq 1} \frac{(-1)^{r} r !}{r^{k}} \sum_{\ell \geq r} S_{2}(\ell, r) \frac{(-t)^{\ell}}{\ell !}=\sum_{r \geq 1} \sum_{\ell \geq r} \frac{(-1)^{r+\ell} r !}{r^{k}} S_{2}(\ell, r) \frac{t^{\ell}}{\ell !} \\
& =\sum_{\ell \geq 1} \sum_{r=1}^{\ell} \frac{(-1)^{r+\ell} r !}{r^{k}} S_{2}(\ell, r) \frac{t^{\ell}}{\ell !},
\end{aligned}
$$

where $S_{2}(n, k)$ is the Stirling number of the second kind, which is defined by the identity $x^{n}=\sum_{k=0}^{n} S_{2}(n, k)(x)_{k}$ with $(x)_{0}=1$ and $(x)_{k}=x(x-1) \cdots(x-k+1)$. This shows

$$
\frac{1}{t} L i_{k}\left(1-e^{-t}\right)=\sum_{\ell \geq 0} \sum_{r=1}^{\ell+1} \frac{(-1)^{r+\ell+1} r !}{r^{k}} \frac{S_{2}(\ell+1, r)}{\ell+1} \frac{t^{\ell}}{\ell !}
$$

Thus,

$$
\begin{aligned}
L i_{k}\left(1-e^{1-e^{t}}\right) & =\sum_{r \geq 1} \sum_{\ell \geq r} \frac{(-1)^{r+\ell} r !}{r^{k}} S_{2}(\ell, r) \frac{\left(e^{t}-1\right)^{\ell}}{\ell !} \\
& =\sum_{r \geq 1} \sum_{\ell \geq r} \sum_{m \geq \ell} \frac{(-1)^{r+\ell} r !}{r^{k}} S_{2}(\ell, r) S_{2}(m, \ell) \frac{t^{m}}{m !} \\
& =\sum_{m \geq 1} \sum_{r=1}^{m} \sum_{\ell=r}^{m} \frac{(-1)^{r+\ell} r !}{r^{k}} S_{2}(\ell, r) S_{2}(m, \ell) \frac{t^{m}}{m !},
\end{aligned}
$$

which implies that

$$
\frac{1}{t} L i_{k}\left(1-e^{1-e^{t}}\right)=\sum_{m \geq 0} \sum_{r=1}^{m+1} \sum_{\ell=r}^{m+1} \frac{(-1)^{r+\ell} r !}{r^{k}} S_{2}(\ell, r) S_{2}(m+1, \ell) \frac{t^{m}}{(m+1) !} .
$$

Now, we are ready to present several formulas for the $n$th poly-Bernoulli polynomials of the second kind.

Theorem 2.1 For all $n \geq 1$,

$$
b_{n}^{(k)}(x)=\sum_{m=0}^{n} \sum_{j=m}^{n} \sum_{r=1}^{j-m+1 j-m+1} \sum_{\ell=r}^{j} \frac{(-1)^{r+\ell}}{j-m+1} \frac{r !}{r^{k}}\left(\begin{array}{c}
n-1 \\
j-1
\end{array}\right)\left(\begin{array}{c}
j \\
m
\end{array}\right) S_{2}(\ell, r) S_{2}(j-m+1, \ell) B_{n-j}^{(n)} x^{m} .
$$

Proof Since $x^{n} \sim(1, t)$ and $\frac{t}{L i_{k}\left(1-e^{1-e^{t}}\right)} b_{n}^{(k)}(x) \sim\left(1, e^{t}-1\right)$ (see (1.6)), we obtain

$$
\begin{aligned}
\frac{t}{L i_{k}\left(1-e^{1-e^{t}}\right)} b_{n}^{(k)}(x) & =x\left(\frac{t}{e^{t}-1}\right)^{n} x^{n-1}=x\left(\sum_{j \geq 0} B_{j}^{(n)} \frac{t^{j}}{j !}\right) x^{n-1} \\
& =x \sum_{j=0}^{n-1}\left(\begin{array}{c}
n-1 \\
j
\end{array}\right) B_{j}^{(n)} x^{n-1-j}=\sum_{j=1}^{n}\left(\begin{array}{c}
n-1 \\
j-1
\end{array}\right) B_{n-j}^{(n)} x^{j} .
\end{aligned}
$$


Thus, by (2.3) we have

$$
\begin{aligned}
b_{n}^{(k)}(x) & =\sum_{j=1}^{n}\left(\begin{array}{c}
n-1 \\
j-1
\end{array}\right) B_{n-j}^{(n)} \frac{L i_{k}\left(1-e^{1-e^{t}}\right)}{t} x^{j} \\
& =\sum_{j=1}^{n}\left(\begin{array}{c}
n-1 \\
j-1
\end{array}\right) B_{n-j}^{(n)}\left(\sum_{m=0}^{j} \sum_{r=1}^{m+1} \sum_{\ell=r}^{m+1} \frac{(-1)^{r+\ell} r !}{r^{k}} S_{2}(\ell, r) S_{2}(m+1, \ell) \frac{t^{m}}{(m+1) !}\right) x^{j} \\
& =\sum_{j=1}^{n} \sum_{m=0}^{j} \sum_{r=1}^{m+1} \sum_{\ell=r}^{m+1} \frac{(-1)^{r+\ell}}{m+1} \frac{r !}{r^{k}}\left(\begin{array}{c}
n-1 \\
j-1
\end{array}\right)\left(\begin{array}{c}
j \\
m
\end{array}\right) S_{2}(\ell, r) S_{2}(m+1, \ell) B_{n-j}^{(n)} x^{j-m} \\
& =\sum_{j=1}^{n} \sum_{m=0}^{j} \sum_{r=1}^{j-m+1} \sum_{\ell=r}^{j-m+1} \frac{(-1)^{r+\ell}}{j-m+1} \frac{r !}{r^{k}}\left(\begin{array}{c}
n-1 \\
j-1
\end{array}\right)\left(\begin{array}{c}
j \\
m
\end{array}\right) S_{2}(\ell, r) S_{2}(j-m+1, \ell) B_{n-j}^{(n)} x^{m},
\end{aligned}
$$

which completes the proof.

Let $S_{1}(n, k)$ be the Stirling number of the first kind, which is defined by the identity $(x)_{n}=$ $\sum_{j=0}^{n} S_{1}(n, k) x^{k}$. Now, we are ready to present our second explicit formula.

Theorem 2.2 For all $n \geq 0$,

$$
b_{n}^{(k)}(x)=\sum_{m=0}^{n} \sum_{j=m}^{n} \sum_{r=1}^{j-m+1} \sum_{\ell=r}^{j-m+1} \frac{(-1)^{r+\ell}}{j-m+1} \frac{r !}{r^{k}}\left(\begin{array}{c}
j \\
m
\end{array}\right) S_{1}(n, j) S_{2}(\ell, r) S_{2}(j-m+1, \ell) x^{m}
$$

Proof Note that $(x)_{n}=\sum_{j=0}^{n} S_{1}(n, j) x^{j} \sim\left(1, e^{t}-1\right)$. So, by (1.6) we have $\frac{t}{L i_{k}\left(1-e^{1-e^{t}}\right)} b_{n}^{(k)}(x) \sim$ $\left(1, e^{t}-1\right)$, which implies that

$$
b_{n}^{(k)}(x)=\sum_{j=0}^{n} S_{1}(n, j) \frac{L i_{k}\left(1-e^{1-e^{t}}\right)}{t} x^{j}
$$

Thus, by (2.3) and using the arguments in the proof of Theorem 2.1, we obtain the required formula.

For the next explicit formula, we use the conjugation representation, namely (1.5).

Theorem 2.3 For all $n \geq 0$,

$$
b_{n}^{(k)}(x)=b_{n}^{(k)}+\sum_{j=1}^{n} \frac{1}{j}\left(\sum_{m=j-1}^{n-1} \sum_{r=1}^{n-m} \frac{(-1)^{r+n-m} r !}{r^{k}}\left(\begin{array}{c}
n \\
m
\end{array}\right) S_{1}(m, j-1) S_{2}(n-m, r)\right) x^{j} .
$$

Proof By (1.5) and (1.6), we have $b_{n}^{(k)}(x)=\sum_{j=0}^{n} c_{n, j} x^{j}$, where

$$
j ! c_{n, j}=\left\langle(g(\bar{f}(t)))^{-1} \bar{f}^{j}(t) \mid x^{n}\right\rangle=\left\langle\frac{L i_{k}\left(1-e^{-t}\right)}{\log (1+t)}(\log (1+t))^{j} \mid x^{n}\right\rangle .
$$


If $j=0$, then $c_{n, 0}=b_{n}^{(k)}$. Thus, assume now that $1 \leq j \leq n$. So

$$
\begin{aligned}
j ! c_{n, j} & =\left\langle L i_{k}\left(1-e^{-t}\right)(\log (1+t))^{j-1} \mid x^{n}\right\rangle \\
& =\left\langle L i_{k}\left(1-e^{-t}\right) \mid(j-1) ! \sum_{m \geq j-1} S_{1}(m, j-1) \frac{t^{m}}{m !} x^{n}\right\rangle \\
& =(j-1) ! \sum_{m=j-1}^{n}\left(\begin{array}{c}
n \\
m
\end{array}\right) S_{1}(m, j-1)\left\langle L i_{k}\left(1-e^{-t}\right) \mid x^{n-m}\right\rangle,
\end{aligned}
$$

which, by (2.1), implies that

$$
\begin{aligned}
j ! c_{n, j} & =(j-1) ! \sum_{m=j-1}^{n}\left(\begin{array}{c}
n \\
m
\end{array}\right) S_{1}(m, j-1)\left\langle\sum_{\ell \geq 1} \sum_{r=1}^{\ell} \frac{(-1)^{r+\ell} r !}{r^{k}} S_{2}(\ell, r) \frac{t^{\ell}}{\ell !} \mid x^{n-m}\right\rangle \\
& =(j-1) ! \sum_{m=j-1}^{n}\left(\begin{array}{c}
n \\
m
\end{array}\right) S_{1}(m, j-1)\left(\sum_{r=1}^{n-m} \frac{(-1)^{r+n-m} r !}{r^{k}} S_{2}(n-m, r)\right),
\end{aligned}
$$

which completes the proof.

In order to state our next formula, we recall that $b_{n}(x)=b_{n}^{(1)}(x)$ is the Bernoulli polynomial of the second kind, which is given by the generating function $\frac{t}{\log (1+t)}(1+t)^{x}=$ $\sum_{n \geq 0} b_{n}(x) \frac{t^{n}}{n !}$.

Theorem 2.4 For all $n \geq 0$,

$$
b_{n}^{(k)}(x)=\frac{1}{n+1} \sum_{j=0}^{n}\left(\begin{array}{c}
n+1 \\
j
\end{array}\right)\left(B_{n+1-j}^{(k)}-B_{n+1-j}^{(k)}(-1)\right) b_{\ell}(x),
$$

where $B_{n}^{(k)}(x)$ is the nth poly-Bernoulli polynomial.

Proof From the definitions, we have

$$
\begin{aligned}
b_{n}^{(k)}(y) & =\left\langle\sum_{\ell \geq 0} b_{\ell}^{(k)}(y) \frac{t^{\ell}}{\ell !} \mid x^{n}\right\rangle=\left\langle\frac{L i_{k}\left(1-e^{-t}\right)}{\log (1+t)}(1+t)^{y} \mid x^{n}\right\rangle \\
& =\left\langle\frac{e^{-t}-1}{-t} \frac{L i_{k}\left(1-e^{-t}\right)}{1-e^{-t}} \frac{t}{\log (1+t)}(1+t)^{y} \mid x^{n}\right\rangle \\
& =\left\langle\frac{e^{-t}-1}{-t} \frac{L i_{k}\left(1-e^{-t}\right)}{1-e^{-t}} \mid \frac{t}{\log (1+t)}(1+t)^{y} x^{n}\right\rangle \\
& =\left\langle\frac{e^{-t}-1}{-t} \frac{L i_{k}\left(1-e^{-t}\right)}{1-e^{-t}} \mid \sum_{\ell \geq 0} b_{\ell}(y) \frac{t^{\ell}}{\ell !} x^{n}\right\rangle .
\end{aligned}
$$

Since $B_{n}^{(k)}(x)$ is the poly-Bernoulli polynomial given by the generating function $\frac{L i_{k}\left(1-e^{-t}\right)}{1-e^{-t}} \times$ $e^{x t}=\sum_{n \geq 0} B_{n}^{(k)}(x) \frac{t^{n}}{n !}$, we have $\frac{L i_{k}\left(1-e^{-t}\right)}{1-e^{-t}} x^{n}=B_{n}^{(k)}(x)$ and $\frac{d}{d x} B_{n}^{(k)}(x)=n B_{n-1}^{(k)}(x)$. Thus $b_{n}^{(k)}(y)=$ $\sum_{\ell=0}^{n}\left(\begin{array}{l}n \\ \ell\end{array}\right) b_{\ell}(y)\left\langle\frac{e^{-t}-1}{-t} \mid B_{n-\ell}^{(k)}(x)\right\rangle$. By the fact that $\langle f(a t) \mid p(x)\rangle=\langle f(t) \mid p(a x)\rangle$ for constant $a$ (see 
Proposition 2.1.11 in [19]), we obtain

$$
b_{n}^{(k)}(y)=\sum_{\ell=0}^{n}\left(\begin{array}{l}
n \\
\ell
\end{array}\right) b_{\ell}(y)\left\langle\frac{e^{t}-1}{t} \mid B_{n-\ell}^{(k)}(-x)\right\rangle .
$$

Note that $\left\langle\frac{e^{t}-1}{t} \mid B_{n-\ell}^{(k)}(-x)\right\rangle=\int_{0}^{1} B_{n-\ell}^{(k)}(-u) d u=\frac{1}{n+1-\ell}\left(B_{n+1-\ell}^{(k)}-B_{n+1-\ell}^{(k)}(-1)\right)$, which leads to

$$
\begin{aligned}
b_{n}^{(k)}(y) & =\sum_{\ell=0}^{n}\left(\begin{array}{l}
n \\
\ell
\end{array}\right) b_{\ell}(y) \frac{1}{n+1-\ell}\left(B_{n+1-\ell}^{(k)}-B_{n+1-\ell}^{(k)}(-1)\right) \\
& =\frac{1}{n+1} \sum_{j=0}^{n}\left(\begin{array}{c}
n+1 \\
j
\end{array}\right)\left(B_{n+1-j}^{(k)}-B_{n+1-j}^{(k)}(-1)\right) b_{\ell}(y),
\end{aligned}
$$

which completes the proof.

Theorem 2.5 For all $n \geq 0$,

$$
b_{n}^{(k)}(x)=\sum_{m=0}^{n}\left(\begin{array}{l}
n \\
m
\end{array}\right)\left[\sum_{r=1}^{m+1}(-1)^{r+m+1} \frac{r ! S_{2}(m+1, r)}{r^{k}(m+1)}\right] b_{n-m}(x)
$$

Proof By using a similar argument as in the proof of Theorem 2.4, we obtain

$$
\begin{aligned}
b_{n}^{(k)}(y) & =\left\langle\frac{L i_{k}\left(1-e^{-t}\right)}{t} \frac{t}{\log (1+t)}(1+t)^{y} \mid x^{n}\right\rangle=\left\langle\frac{L i_{k}\left(1-e^{-t}\right)}{t} \mid \sum_{m \geq 0} b_{m}(y) \frac{t^{m}}{m !} x^{n}\right\rangle \\
& =\sum_{m=0}^{n}\left(\begin{array}{l}
n \\
m
\end{array}\right) b_{m}(y)\left\langle\frac{L i_{k}\left(1-e^{-t}\right)}{t} \mid x^{n-m}\right\rangle,
\end{aligned}
$$

which, by (2.2), gives

$$
\begin{aligned}
b_{n}^{(k)}(y) & =\sum_{m=0}^{n}\left(\begin{array}{l}
n \\
m
\end{array}\right) b_{m}(y)\left\langle\sum_{\ell \geq 0} \sum_{r=1}^{\ell+1} \frac{(-1)^{r+\ell+1} r !}{r^{k}} \frac{S_{2}(\ell+1, r)}{\ell+1} \frac{t^{\ell}}{\ell !} \mid x^{n-m}\right\rangle \\
& =\sum_{m=0}^{n}\left(\begin{array}{l}
n \\
m
\end{array}\right) b_{m}(y)\left(\sum_{r=1}^{n-m+1} \frac{(-1)^{r+n-m+1} r !}{r^{k}} \frac{S_{2}(n-m+1, r)}{n-m+1}\right) \\
& =\sum_{m=0}^{n}\left(\begin{array}{l}
n \\
m
\end{array}\right)\left[\sum_{r=1}^{m+1}(-1)^{r+m+1} \frac{r ! S_{2}(m+1, r)}{r^{k}(m+1)}\right] b_{n-m}(y),
\end{aligned}
$$

as required.

Note that the statement of Theorem 2.5 has been obtained in Theorem 2.2 of [12].

\section{Recurrence relations}

By (1.6) we have $b_{n}^{(k)}(x) \sim\left(\frac{t}{L i_{k}\left(1-e^{1-e^{t}}\right)}, e^{t}-1\right)$ with $P_{n}(x)=\frac{t}{L i_{k}\left(1-e^{1-e^{t}}\right)} b_{n}^{(k)}(x)=(x)_{n}=x(x-$ 1) $\cdots(x-n+1) \sim\left(1, e^{t}-1\right)$. Thus,

$$
b_{n}^{(k)}(x+y)=\sum_{j=0}^{n}\left(\begin{array}{l}
n \\
j
\end{array}\right) b_{j}^{(k)}(x)(y)_{n-j}
$$


The aim of this section is to derive recurrence relations for the poly-Bernoulli polynomials of the second kind. As first trivial recurrence, by using the fact that if $S_{n}(x) \sim(g(t), f(t))$ then $f(t) S_{n}(x)=n S_{n-1}(x)$, we derive that $\left(e^{t}-1\right) b_{n}^{(k)}(x)=n b_{n-1}^{(k)}(x)$, and hence $b_{n}^{(k)}(x+1)=$ $b_{n}^{(k)}(x)+n b_{n-1}^{(k)}(x)$. Our next results establish other types of recurrence relations.

Theorem 3.1 For all $n \geq 0$,

$$
\begin{aligned}
b_{n+1}^{(k)}(x)= & x b_{n}^{(k)}(x-1) \\
& +\sum_{j=0}^{n} \sum_{\ell=0}^{j+1} \sum_{m=0}^{j+1-\ell} \frac{1}{m}\left(\begin{array}{c}
j \\
m-1
\end{array}\right) S_{1}(n, j) S_{2}(j+1-m, \ell)\left(B_{\ell}^{(k-1)}(-1) x^{m}-b_{\ell}^{(k)}(x-1)^{m}\right) .
\end{aligned}
$$

Proof It is well known that if $S_{n}(x) \sim(g(t), f(t))$ then $S_{n+1}(x)=\left(x-\frac{g^{\prime}(t)}{g(t)}\right) \frac{1}{f^{\prime}(t)} S_{n}(x)$. Hence, by (1.6), we have

$$
b_{n+1}^{(k)}(x)=x b_{n}^{(k)}(x-1)-e^{-t} \frac{g^{\prime}(t)}{g(t)} b_{n}^{(k)}(x)
$$

with

$$
\frac{g^{\prime}(t)}{g(t)}=(\log (g(t)))^{\prime}=\left(\log t-\log L i_{k}\left(1-e^{1-e^{t}}\right)\right)^{\prime}=\frac{1}{t}\left(1-\frac{t e^{t} e^{1-e^{t}} L i_{k-1}\left(1-e^{1-e^{t}}\right)}{\left(1-e^{1-e^{t}}\right) L i_{k}\left(1-e^{1-e^{t}}\right)}\right)
$$

where $1-\frac{t e^{t} e^{1-e^{t}} L i_{k-1}\left(1-e^{1-e^{t}}\right)}{\left(1-e^{1-e^{t}}\right) L i_{k}\left(1-e^{1-e^{t}}\right)}$ has order at least one. Thus, by (2.4), we get

$$
\begin{aligned}
-e^{-t} \frac{g^{\prime}(t)}{g(t)} b_{n}^{(k)}(x) & =\frac{-e^{-t}}{t}\left(1-\frac{t e^{t} e^{1-e^{t}} L i_{k-1}\left(1-e^{1-e^{t}}\right)}{\left(1-e^{1-e^{t}}\right) L i_{k}\left(1-e^{1-e^{t}}\right)}\right) \sum_{j=0}^{n} S_{1}(n, j) \frac{L i_{k}\left(1-e^{1-e^{t}}\right)}{t} x^{j} \\
& =-\sum_{j=0}^{n} \frac{S_{1}(n, j)}{j+1}\left(\frac{e^{-t} L i_{k}\left(1-e^{1-e^{t}}\right)}{\log \left(1+e^{t}-1\right)}-\frac{e^{1-e^{t}} L i_{k-1}\left(1-e^{1-e^{t}}\right)}{\left(1-e^{1-e^{t}}\right)}\right) x^{j+1} \\
& =-\sum_{j=0}^{n} \frac{S_{1}(n, j)}{j+1}\left(e^{-t} \sum_{\ell \geq 0} b_{\ell}^{(k)} \frac{\left(e^{t}-1\right)^{\ell}}{\ell !}-\sum_{\ell \geq 0} B_{\ell}^{(k-1)}(-1) \frac{\left(e^{t}-1\right)^{\ell}}{\ell !}\right) x^{j+1},
\end{aligned}
$$

where

$$
\begin{aligned}
e^{-t} & \sum_{\ell \geq 0} b_{\ell}^{(k)} \frac{\left(e^{t}-1\right)^{\ell}}{\ell !} x^{j+1} \\
& =e^{-t} \sum_{\ell=0}^{j+1} b_{\ell}^{(k)} \sum_{m=\ell}^{j+1} S_{2}(m, \ell) \frac{t^{m}}{m !} x^{j+1}=e^{-t} \sum_{\ell=0}^{j+1} \sum_{m=\ell}^{j+1}\left(\begin{array}{c}
j+1 \\
m
\end{array}\right) b_{\ell}^{(k)} S_{2}(m, \ell) x^{j+1-m} \\
& =e^{-t} \sum_{\ell=0}^{j+1} \sum_{m=0}^{j+1-\ell}\left(\begin{array}{c}
j+1 \\
m
\end{array}\right) b_{\ell}^{(k)} S_{2}(j+1-m, \ell) x^{m} \\
& =\sum_{\ell=0}^{j+1} \sum_{m=0}^{j+1-\ell}\left(\begin{array}{c}
j+1 \\
m
\end{array}\right) b_{\ell}^{(k)} S_{2}(j+1-m, \ell)(x-1)^{m}
\end{aligned}
$$


and

$$
\begin{aligned}
\sum_{\ell \geq 0} B_{\ell}^{(k-1)}(-1) \frac{\left(e^{t}-1\right)^{\ell}}{\ell !} x^{j+1} & =\sum_{\ell=0}^{j+1} B_{\ell}^{(k-1)}(-1) \sum_{m=\ell}^{j+1} S_{2}(m, \ell) \frac{t^{m}}{m !} x^{j+1} \\
& =\sum_{\ell=0}^{j+1} \sum_{m=\ell}^{j+1}\left(\begin{array}{c}
j+1 \\
m
\end{array}\right) S_{2}(m, \ell) B_{\ell}^{(k-1)}(-1) x^{j+1-m} \\
& =\sum_{\ell=0}^{j+1} \sum_{m=0}^{j+1-\ell}\left(\begin{array}{c}
j+1 \\
m
\end{array}\right) S_{2}(j+1-m, \ell) B_{\ell}^{(k-1)}(-1) x^{m} .
\end{aligned}
$$

Thus,

$$
\begin{aligned}
b_{n+1}^{(k)}(x)= & x b_{n}^{(k)}(x-1) \\
& +\sum_{j=0}^{n} \frac{S_{1}(n, j)}{j+1} \sum_{\ell=0}^{j+1} \sum_{m=0}^{j+1-\ell}\left(\begin{array}{c}
j+1 \\
m
\end{array}\right) S_{2}(j+1-m, \ell)\left(B_{\ell}^{(k-1)}(-1) x^{m}-b_{\ell}^{(k)}(x-1)^{m}\right) \\
= & x b_{n}^{(k)}(x-1) \\
& +\sum_{j=0}^{n} \sum_{\ell=0}^{j+1} \sum_{m=0}^{j+1-\ell} \frac{1}{m}\left(\begin{array}{c}
j \\
m-1
\end{array}\right) S_{1}(n, j) S_{2}(j+1-m, \ell)\left(B_{\ell}^{(k-1)}(-1) x^{m}-b_{\ell}^{(k)}(x-1)^{m}\right),
\end{aligned}
$$

which completes the proof.

Theorem 3.2 For all $n \geq 0, \frac{d}{d x} b_{n}^{(k)}(x)=n ! \sum_{\ell=0}^{n-1} \frac{(-1)^{n-1-\ell}}{\ell !(n-\ell)} b_{\ell}^{(k)}(x)$.

Proof We proceed in the proof by using the fact that if $S_{n}(x) \sim(g(t), f(t))$ then

$$
\frac{d}{d x} S_{n}(x)=\sum_{\ell=0}^{n-1}\left(\begin{array}{l}
n \\
\ell
\end{array}\right)\left\langle\bar{f}(t)\left|x^{n-\ell}\right| S_{\ell}(x) .\right.
$$

By (1.6), we have $\left\langle\bar{f}(t) \mid x^{n-\ell}\right\rangle=\left\langle\log (1+t) \mid x^{n-\ell}\right\rangle$, which leads to

$$
\left\langle\bar{f}(t) \mid x^{n-\ell}\right\rangle=\left\langle\sum_{m \geq 1}(-1)^{m-1}(m-1) ! \frac{t^{m}}{m !} \mid x^{n-\ell}\right\rangle=(-1)^{n-1-\ell}(n-1-\ell) ! .
$$

Thus $\frac{d}{d x} b_{n}^{(k)}(x)=n ! \sum_{\ell=0}^{n-1} \frac{(-1)^{n-1-\ell} \ell !(n-\ell)}{(k)} b_{\ell}^{(x)}(x)$, as required.

Theorem 3.3 For all $n \geq 1$,

$$
b_{n}^{(k)}(x)=x b_{n-1}^{(k)}(x-1)+\frac{1}{n} \sum_{\ell=0}^{n}\left(\begin{array}{l}
n \\
\ell
\end{array}\right)\left(B_{\ell}^{(k-1)}(-1) b_{n-\ell}(x)-b_{\ell}^{(k)} b_{n-\ell}(x-1)\right) .
$$

Proof Let $n \geq 1$. Then (1.6), we have

$$
\begin{aligned}
b_{n}^{(k)}(y) & =\left\langle\frac{L i_{k}\left(1-e^{-t}\right)}{\log (1+t)}(1+t)^{y} \mid x^{n}\right\rangle=\left\langle\frac{d}{d t}\left[\frac{L i_{k}\left(1-e^{-t}\right)}{\log (1+t)}(1+t)^{y}\right] \mid x^{n-1}\right\rangle \\
& =\left\langle\frac{L i_{k}\left(1-e^{-t}\right)}{\log (1+t)} \frac{d}{d t}\left[(1+t)^{y}\right] \mid x^{n-1}\right\rangle+\left\langle\frac{d}{d t}\left[\frac{L i_{k}\left(1-e^{-t}\right)}{\log (1+t)}\right](1+t)^{y} \mid x^{n-1}\right\rangle .
\end{aligned}
$$


The first term in (3.1) is given by

$$
\left\langle\frac{L i_{k}\left(1-e^{-t}\right)}{\log (1+t)} \frac{d}{d t}\left[(1+t)^{y}\right] \mid x^{n-1}\right\rangle=y\left\langle\frac{L i_{k}\left(1-e^{-t}\right)}{\log (1+t)}(1+t)^{y-1} \mid x^{n-1}\right\rangle=y b_{n-1}^{(k)}(y-1) .
$$

For the second term in (3.1), we note that

$$
\begin{aligned}
\frac{d}{d t}\left[\frac{L i_{k}\left(1-e^{-t}\right)}{\log (1+t)}\right](1+t)^{y} & =\frac{1}{t} \frac{t}{\log (1+t)}\left(\frac{L i_{k-1}\left(1-e^{-t}\right)}{1-e^{-t}} e^{-t}-\frac{L i_{k}\left(1-e^{-t}\right)}{\log (1+t)} \frac{1}{1+t}\right)(1+t)^{y} \\
& =\frac{1}{t}\left(\frac{t(1+t)^{y}}{\log (1+t)} \frac{L i_{k-1}\left(1-e^{-t}\right)}{1-e^{-t}} e^{-t}-\frac{t(1+t)^{y-1}}{\log (1+t)} \frac{L i_{k}\left(1-e^{-t}\right)}{\log (1+t)}\right),
\end{aligned}
$$

which has order at least zero. So, the second term in (3.1) is given by

$$
\begin{aligned}
\left\langle\frac{d}{d t}\right. & {\left[\frac{L i_{k}\left(1-e^{-t}\right)}{\log (1+t)}\right](1+t)^{y}\left|x^{n-1}\right\rangle } \\
= & \frac{1}{n}\left(\left\langle\frac{t}{\log (1+t)}(1+t)^{y} \mid \frac{L i_{k-1}\left(1-e^{-t}\right)}{1-e^{-t}} e^{-t} x^{n}\right\rangle\right. \\
& \left.-\left\langle\frac{t}{\log (1+t)}(1+t)^{y-1} \mid \frac{L i_{k}\left(1-e^{-t}\right)}{\log (1+t)} x^{n}\right\rangle\right) \\
= & \frac{1}{n}\left(\left\langle\frac{t}{\log (1+t)}(1+t)^{y} \mid \sum_{\ell \geq 0} B_{\ell}^{(k-1)}(-1) \frac{t^{\ell}}{\ell !} x^{n}\right\rangle\right. \\
& \left.-\left\langle\frac{t}{\log (1+t)}(1+t)^{y-1} \mid \sum_{\ell \geq 0} b_{\ell}^{(k)} \frac{t^{\ell}}{\ell !} x^{n}\right\rangle\right) \\
= & \frac{1}{n}\left(\sum_{\ell=0}^{n}\left(\begin{array}{l}
n \\
\ell
\end{array}\right) B_{\ell}^{(k-1)}(-1)\left\langle\frac{t}{\log (1+t)}(1+t)^{y} \mid x^{n-\ell}\right\rangle\right. \\
& \left.-\sum_{\ell=0}^{n}\left(\begin{array}{l}
n \\
\ell
\end{array}\right) b_{\ell}^{(k)}\left\langle\frac{t}{\log (1+t)}(1+t)^{y-1} \mid x^{n-\ell}\right\rangle\right) \\
= & \frac{1}{n}\left(\sum_{\ell=0}^{n}\left(\begin{array}{l}
n \\
\ell
\end{array}\right) B_{\ell}^{(k-1)}(-1) b_{n-\ell}(y)-\sum_{\ell=0}^{n}\left(\begin{array}{l}
n \\
\ell
\end{array}\right) b_{\ell}^{(k)} b_{n-\ell}(y-1)\right) \\
= & \frac{1}{n} \sum_{\ell=0}^{n}\left(\begin{array}{l}
n \\
\ell
\end{array}\right)\left(B_{\ell}^{(k-1)}(-1) b_{n-\ell}(y)-b_{\ell}^{(k)} b_{n-\ell}(y-1)\right) .
\end{aligned}
$$

By substituting (3.2) and (3.3) into (3.1), we complete the proof.

\section{Identities}

In this section we present some identities related to poly-Bernoulli numbers of the second kind.

Theorem 4.1 For all $n \geq 0$,

$$
\sum_{\ell=0}^{n}(-1)^{n-\ell}(n-\ell) !\left(\begin{array}{c}
n+1 \\
\ell
\end{array}\right) b_{\ell}^{(k)}=\sum_{m=0}^{n}(-1)^{n-m}\left(\begin{array}{l}
n \\
m
\end{array}\right) B_{m}^{(k-1)}
$$


Proof We compute $A=\left\langle L i_{k}\left(1-e^{-t}\right) \mid x^{n+1}\right\rangle$ in two different ways. On the one hand, by (1.6), it is

$$
\begin{aligned}
A & =\left\langle\frac{L i_{k}\left(1-e^{-t}\right)}{\log (1+t)} \mid \log (1+t) x^{n+1}\right\rangle=\left\langle\frac{L i_{k}\left(1-e^{-t}\right)}{\log (1+t)} \mid \sum_{\ell \geq 1} \frac{(-1)^{\ell-1} t^{\ell}}{\ell} x^{n+1}\right\rangle \\
& =\sum_{\ell=0}^{n}(-1)^{n-\ell}(n-\ell) !\left(\begin{array}{c}
n+1 \\
\ell
\end{array}\right)\left\langle\frac{L i_{k}\left(1-e^{-t}\right)}{\log (1+t)} \mid x^{\ell}\right\rangle \\
& =\sum_{\ell=0}^{n}(-1)^{n-\ell}(n-\ell) !\left(\begin{array}{c}
n+1 \\
\ell
\end{array}\right) b_{\ell}^{(k)} .
\end{aligned}
$$

On the other hand, by (1.6), it is

$$
\begin{aligned}
A & =\left\langle L i_{k}\left(1-e^{-t}\right) \mid x^{n+1}\right\rangle=\left\langle\int_{0}^{t} \frac{d}{d s} L i_{k}\left(1-e^{-s}\right) d s \mid x^{n+1}\right\rangle=\left\langle\int_{0}^{t} e^{-s} \frac{L i_{k-1}\left(1-e^{-s}\right)}{1-e^{-s}} d s \mid x^{n+1}\right\rangle \\
& =\left\langle\int_{0}^{t} \sum_{a \geq 0} \frac{(-s)^{a}}{a !} \sum_{m \geq 0} B_{m}^{(k-1)} \frac{s^{m}}{m !} d s \mid x^{n+1}\right\rangle=\left\langle\sum_{\ell \geq 0} \sum_{m=0}^{\ell}(-1)^{\ell-m}\left(\begin{array}{c}
\ell \\
m
\end{array}\right) B_{m}^{(k-1)} \frac{t^{\ell+1}}{(\ell+1) !} \mid x^{n+1}\right\rangle \\
& =\sum_{m=0}^{n}(-1)^{n-m}\left(\begin{array}{c}
n \\
m
\end{array}\right) B_{m}^{(k-1)} .
\end{aligned}
$$

By comparing (4.1) and (4.2), we obtain the required identity.

By using similar techniques as in the proof of Theorem 4.1 with computing

$$
\left\langle\frac{L i_{k}\left(1-e^{-t}\right)}{\log (1+t)}(\log (1+t))^{m} \mid x^{n}\right\rangle
$$

in two different ways, we obtain the following result (we leave the proof as an exercise to the interested reader).

Theorem 4.2 For all $n-1 \geq m \geq 1$,

$$
\begin{aligned}
\sum_{\ell=0}^{n-m}\left(\begin{array}{l}
n \\
\ell
\end{array}\right) S_{1}(n-\ell, m) b_{\ell}^{(k)} \\
=\sum_{\ell=0}^{n-m}\left(\begin{array}{c}
n-1 \\
\ell
\end{array}\right) S_{1}(n-1-\ell, m-1) b_{\ell}^{(k)}(-1) \\
\quad+\frac{1}{n} \sum_{\ell=0}^{n-1-m} \sum_{j=0}^{\ell+1}\left(\begin{array}{c}
n \\
\ell+1
\end{array}\right)\left(\begin{array}{c}
\ell+1 \\
j
\end{array}\right) S_{1}(n-1-\ell, m)\left(b_{\ell+1-j} B_{j}^{(k-1)}(-1)-b_{\ell+1-j}(-1) b_{j}^{(k)}\right) .
\end{aligned}
$$

Let $b_{n}^{(k)}(x)=\sum_{m=0}^{n} c_{n, m}(x)_{m}$. By (1.5), (1.6) and the fact that $(x)_{m} \sim\left(1, e^{t}-1\right)$, we obtain

$$
c_{n, m}=\frac{1}{m !}\left\langle\frac{L i_{k}\left(1-e^{-t}\right)}{\log (1+t)} \mid t^{m} x^{n}\right\rangle=\left(\begin{array}{c}
n \\
m
\end{array}\right)\left\langle\frac{L i_{k}\left(1-e^{-t}\right)}{\log (1+t)} \mid x^{n-m}\right\rangle=\left(\begin{array}{c}
n \\
m
\end{array}\right) b_{n-m}^{(k)},
$$

which leads to the following identity. 
Theorem 4.3 For all $n \geq 0$,

$$
b_{n}^{(k)}(x)=\sum_{m=0}^{n}\left(\begin{array}{c}
n \\
m
\end{array}\right) b_{n-m}^{(k)}(x)_{m} .
$$

Let $\mathbb{B}_{n}^{(s)}(x)$ be the $n$th Bernoulli polynomial of order $s$. Then $\mathbb{B}_{n}^{(s)}(x) \sim\left(\left(\left(e^{t}-1\right) / t\right)^{s}, t\right)$. Also, the Bernoulli numbers of the second kind of order $s$ are given by $\frac{t^{s}}{\log ^{s}(1+t)}=\sum_{j \geq 0} \mathbf{b}_{j}^{(s)} \frac{t_{j !}^{j}}{j !}$ and let $b_{n}^{(k)}(x)=\sum_{m=0}^{n} c_{n, m} \mathbb{B}_{m}^{(s)}(x)$. By (1.5) and (1.6), we obtain

$$
\begin{aligned}
c_{n, m} & =\frac{1}{m !}\left\langle\frac{\frac{t^{s}}{\log (1+t)} \frac{\log (1+t)}{L i_{k}\left(1-e^{-t}\right)}}{\log m}(1+t) \mid x^{n}\right\rangle=\frac{1}{m !}\left\langle\frac{L i_{k}\left(1-e^{-t}\right)}{\log (1+t)} \frac{t^{s}}{\log ^{s}(1+t)} \mid \log ^{m}(1+t) x^{n}\right\rangle \\
& =\frac{1}{m !}\left\langle\frac{L i_{k}\left(1-e^{-t}\right)}{\log (1+t)} \frac{t^{s}}{\log ^{s}(1+t)} \mid m ! \sum_{\ell \geq m} S_{1}(\ell, m) \frac{t^{\ell}}{\ell !} x^{n}\right\rangle \\
& =\sum_{\ell=m}^{n}\left(\begin{array}{l}
n \\
\ell
\end{array}\right) S_{1}(\ell, m)\left\langle\frac{L i_{k}\left(1-e^{-t}\right)}{\log (1+t)} \frac{t^{s}}{\log ^{s}(1+t)} \mid x^{n-\ell}\right\rangle \\
& =\sum_{\ell=0}^{n-m}\left(\begin{array}{l}
n \\
\ell
\end{array}\right) S_{1}(n-\ell, m)\left\langle\frac{L i_{k}\left(1-e^{-t}\right)}{\log (1+t)} \mid \frac{t^{s}}{\log ^{s}(1+t)} x^{\ell}\right\rangle \\
& =\sum_{\ell=0}^{n-m}\left(\begin{array}{l}
n \\
\ell
\end{array}\right) S_{1}(n-\ell, m)\left\langle\frac{L i_{k}\left(1-e^{-t}\right)}{\log (1+t)} \mid \sum_{j \geq 0} \mathbf{b}_{j}^{(s)} \frac{t^{j}}{j !} x^{\ell}\right\rangle \\
& =\sum_{\ell=0}^{n-m} \sum_{j=0}^{\ell}\left(\begin{array}{l}
n \\
\ell
\end{array}\right)\left(\begin{array}{l}
\ell \\
j
\end{array}\right) S_{1}(n-\ell, m) \mathbf{b}_{j}^{(s)}\left\langle\frac{L i_{k}\left(1-e^{-t}\right)}{\log (1+t)} \mid x^{\ell-j}\right\rangle \\
& =\sum_{\ell=0}^{n-m} \sum_{j=0}^{\ell}\left(\begin{array}{l}
n \\
\ell
\end{array}\right)\left(\begin{array}{l}
\ell \\
j
\end{array}\right) S_{1}(n-\ell, m) \mathbf{b}_{j}^{(s)} b_{\ell-j}^{(k)},
\end{aligned}
$$

which gives the following identity.

Theorem 4.4 For all $n \geq 0$,

$$
b_{n}^{(k)}(x)=\sum_{m=0}^{n}\left(\sum_{\ell=0}^{n-m} \sum_{j=0}^{\ell}\left(\begin{array}{l}
n \\
\ell
\end{array}\right)\left(\begin{array}{l}
\ell \\
j
\end{array}\right) S_{1}(n-\ell, m) \mathbf{b}_{j}^{(s)} b_{\ell-j}^{(k)}\right) \mathbb{B}_{m}^{(s)}(x) .
$$

Define $H_{n}^{(s)}(\lambda, x)$ to be the $n$th Frobenius-Euler polynomials of order $s$. Note that these polynomial satisfy $H_{n}^{(s)}(\lambda, x) \sim\left(\left(\left(e^{t}-\lambda\right) /(1-\lambda)\right)^{s}, t\right)$. Let $b_{n}^{(k)}(x)=\sum_{m=0}^{n} c_{n, m} H_{m}^{(s)}(\lambda, x)$. By (1.5) and (1.6), we obtain

$$
\begin{aligned}
c_{n, m} & =\frac{1}{m !}\left\langle\frac{\frac{(1+t-\lambda)^{s}}{(1-\lambda)^{s}}}{\frac{\log (1+t)}{L i_{k}\left(1-e^{-t}\right)}} \log ^{m}(1+t) \mid x^{n}\right\rangle=\frac{1}{m !(1-\lambda)^{s}}\left\langle\frac{L i_{k}\left(1-e^{-t}\right)}{\log (1+t)} \log ^{m}(1+t) \mid(1-\lambda+t)^{s} x^{n}\right\rangle \\
& =\frac{1}{m !(1-\lambda)^{s}} \sum_{j=0}^{n-m}\left(\begin{array}{l}
s \\
j
\end{array}\right)(1-\lambda)^{s-j}(n)_{j}\left\langle\frac{L i_{k}\left(1-e^{-t}\right)}{\log (1+t)} \mid \log ^{m}(1+t) x^{n-j}\right\rangle \\
& =\frac{1}{m !(1-\lambda)^{s}} \sum_{j=0}^{n-m}\left(\begin{array}{l}
s \\
j
\end{array}\right)(1-\lambda)^{s-j}(n)_{j}\left\langle\frac{L i_{k}\left(1-e^{-t}\right)}{\log (1+t)} \mid m ! \sum_{\ell \geq m} S_{1}(\ell, m) \frac{t^{\ell}}{\ell !} x^{n-j}\right\rangle
\end{aligned}
$$




$$
\begin{aligned}
& =\sum_{j=0}^{n-m} \sum_{\ell=m}^{n-j}\left(\begin{array}{c}
s \\
j
\end{array}\right)\left(\begin{array}{c}
n-j \\
\ell
\end{array}\right) S_{1}(\ell, m)(1-\lambda)^{-j}(n)_{j}\left\langle\frac{L i_{k}\left(1-e^{-t}\right)}{\log (1+t)} \mid x^{n-j-\ell}\right\rangle \\
& =\sum_{j=0}^{n-m} \sum_{\ell=m}^{n-j}\left(\begin{array}{c}
s \\
j
\end{array}\right)\left(\begin{array}{c}
n-j \\
\ell
\end{array}\right) S_{1}(\ell, m)(1-\lambda)^{-j}(n)_{j} b_{n-j-\ell}^{(k)} \\
& =\sum_{j=0}^{n-m} \sum_{\ell=0}^{n-m-j}\left(\begin{array}{c}
s \\
j
\end{array}\right)\left(\begin{array}{c}
n-j \\
\ell
\end{array}\right) S_{1}(n-j-\ell, m)(1-\lambda)^{-j}(n)_{j} b_{\ell}^{(k)},
\end{aligned}
$$

which gives the following identity.

Theorem 4.5 For all $n \geq 0$,

$$
b_{n}^{(k)}(x)=\sum_{m=0}^{n}\left(\sum_{j=0}^{n-m} \sum_{\ell=0}^{n-m-j}\left(\begin{array}{c}
s \\
j
\end{array}\right)\left(\begin{array}{c}
n-j \\
\ell
\end{array}\right) S_{1}(n-j-\ell, m)(1-\lambda)^{-j}(n)_{j} b_{\ell}^{(k)}\right) H_{m}^{(s)}(\lambda, x) .
$$

\section{Competing interests}

The authors declare that they have no competing interests.

\section{Authors' contributions}

All authors contributed equally to this work. All authors read and approved the final manuscript.

\section{Author details}

'Department of Mathematics, Sogang University, Seoul, South Korea. ${ }^{2}$ Department of Mathematics, Kwangwoon University, Seoul, South Korea. ${ }^{3}$ Department of Mathematics, University of Haifa, Haifa, 31905, Israel. ${ }^{4}$ Institute of Natural Sciences, Far Eastern Federal University, Vladivostok, 690950, Russia.

\section{Acknowledgements}

This work was partially supported by Kwangwoon University in 2014

Received: 19 November 2014 Accepted: 7 January 2015 Published online: 31 January 2015

\section{References}

1. Kaneko, M: Poly-Bernoulli numbers. J. Théor. Nr. Bordx. 9, 221-228 (1997)

2. Kim, DS, Kim, T, Lee, S-H: Poly-Cauchy numbers and polynomials with umbral calculus viewpoint. Int. J. Math. Anal. 7(45-48), 2235-2253 (2013)

3. Kim, T: Higher-order Cauchy of the second kind and poly-Cauchy of the second kind mixed type polynomials. Ars Comb. 115, 435-451 (2014)

4. Zhao, F-Z: Some results for generalized Cauchy numbers. Util. Math. 82, 269-284 (2010)

5. Araci, S, Acikgoz, M: A note on the Frobenius-Euler numbers and polynomials associated with Bernstein polynomials. Adv. Stud. Contemp. Math. (Kyungshang) 22(3), 399-406 (2012)

6. Gzyl, H: Hamilton Flows and Evolution Semigroups. Pitman Research Notes in Mathematics Series, vol. 239 (1990)

7. Jolany, H, Sharifi, H, Alikelaye, RE: Some results for the Apostol-Genocchi polynomials of higher order. Bull. Malays. Math. Soc. 36(2), 465-479 (2013)

8. Qi, F: An integral representation, complete monotonicity, and inequalities of Cauchy numbers of the second kind. J. Number Theory 144, 244-255 (2014)

9. Nemes, G: An asymptotic expansion for the Bernoulli numbers of the second kind. J. Integer Seq. 14(4), Article 11.4.8 (2011)

10. Kim, T: Identities involving Laguerre polynomials derived from umbral calculus. Russ. J. Math. Phys. 21(1), 36-45 (2014)

11. Zachos, CK: Umbral deformations on discrete space-time. Int. J. Mod. Phys. A 23(13), 2005-2014 (2008)

12. Kim, T, Kwon, H-I, Lee, S-H, Seo, J-J: A note on poly-Bernoulli numbers and polynomials of the second kind. Adv. Differ. Equ. 2014, Article ID 219 (2014)

13. Biedenharn, LC, Gustafson, RA, Lohe, MA, Louck, JD, Milne, SC: Special functions and group theory in theoretical physics. In: Special Functions: Group Theoretical Aspects and Applications. Math. Appl., pp. 129-162. Reidel, Dordrecht (1984)

14. Dattoli, G, Levi, D, Winternitz, P: Heisenberg algebra, umbral calculus and orthogonal polynomials. J. Math. Phys. 49(5), 053509 (2008)

15. Blasiak, P, Dattoli, G, Horzela, A, Penson, KA: Representations of monomiality principle with Sheffer-type polynomials and boson normal ordering. Phys. Lett. A 352, 7-12 (2006)

16. Kim, DS, Kim, T: Applications of umbral calculus associated with $p$-adic invariant integrals on $\mathbb{Z}_{p}$. Abstr. Appl. Anal. 2012, Article ID 865721 (2012) 
17. Kim, DS, Kim, T: Some identities of Frobenius-Euler polynomials arising from umbral calculus. Adv. Differ. Equ. 2012, Article ID 196 (2012)

18. Roman, S: More on the umbral calculus, with emphasis on the $q$-umbral calculus. J. Math. Anal. Appl. 107, 222-254 (1985)

19. Roman, S: The Umbral Calculus. Dover, New York (2005)

Submit your manuscript to a SpringerOpen ${ }^{\circ}$ journal and benefit from:

- Convenient online submission

- Rigorous peer review

- Immediate publication on acceptance

- Open access: articles freely available online

- High visibility within the field

- Retaining the copyright to your article

Submit your next manuscript at $\gg$ springeropen.com 